\title{
Manganese Functionalized Silicate Nanoparticles as a Fenton-Type Catalyst for Water Purification by Advanced Oxidation Processes (AOP)
}

\author{
Nataša Novak Tušar,* Darja Maučec, Mojca Rangus, Iztok Arčon, Matjaž Mazaj, \\ Magda Cotman, Albin Pintar, and Venčeslav Kaučič
}

Wet hydrogen peroxide catalytic oxidation (WHPCO) is one of the most important industrially applicable advanced oxidation processes (AOPs) for the decomposition of organic pollutants in water. It is demonstrated that manganese functionalized silicate nanoparticles with interparticle porosity act as a superior Fenton-type nanocatalyst in WHPCO as they can decompose $80 \%$ of a test organic compound in 30 minutes at neutral pH and room temperature. By using X-ray absorption spectroscopic techniques it is also shown that the superior activity of the nanocatalyst can be attributed uniquely to framework manganese, which decomposes $\mathrm{H}_{2} \mathrm{O}_{2}$ to reactive hydroxyls and, unlike manganese in $\mathrm{Mn}_{3} \mathrm{O}_{4}$ or $\mathrm{Mn}_{2} \mathrm{O}_{3}$ nanoparticles, does not promote the simultaneous decomposition of hydrogen peroxide. The presented material thus introduces a new family of Fenton nanocatalysts, which are environmentally friendly, cost-effective, and possess superior efficiency for the decomposition of $\mathrm{H}_{2} \mathrm{O}_{2}$ to reactive hydroxyls (AOP), which in turn readily decompose organic pollutants dissolved in water.

\section{Introduction}

Industry generates increasing amounts of wastewater which is contaminated with toxic and hazardous compounds that cause severe problems for the environment. Organic compounds (dyes, pesticides, pharmaceuticals, endocrine disrupting chemicals, etc.) are one of the most important groups of pollutants present in water. ${ }^{[1]}$ The development of powerful and practical treatments of organic compounds in drinking- and waste-water, has attracted world-wide attention over past two decades. ${ }^{[2]}$ Advanced oxidation processes (AOPs) have recently been intensively investigated because they can decompose organic pollutants to inorganic compounds such as $\mathrm{H}_{2} \mathrm{O}, \mathrm{CO}_{2}$ and inorganic

Dr. N. N. Tušar, D. Maučec, M. Rangus, Dr. M. Mazaj, Dr. M. Cotman, Prof. A. Pintar, Prof. V. Kaučič

National Institute of Chemistry

Hajdrihova 19, 1000, Ljubljana, Slovenia

E-mail: natasa.novak@ki.si

Dr. N. N. Tušar, Prof. I. Arčon

University of Nova Gorica

Vipavska 13, 5000, Nova Gorica, Slovenia

DOI: 10.1002/adfm.201102361 salts. ${ }^{[3]}$ The main mechanism of AOPs is the generation of highly reactive free hydroxyl radicals (electrophiles), which react rapidly and nonselectively with nearly all electron-rich organic compounds.

A large number of methods are classified under the broad definition of AOPs. Most AOPs use a combination of strong oxidizing agents (e.g. $\mathrm{H}_{2} \mathrm{O}_{2}, \mathrm{O}_{3}$ ) with catalysts (e.g. transition metals) and/or irradiation (e.g., ultraviolet and visible light). Wet hydrogen peroxide catalytic oxidation (WHPCO) is one of the AOPs with the advantage that it does not require costly reactors, which is important for industrial application. ${ }^{[4]}$ The catalyst currently used for WHPCO in commercial applications is a homogeneous Fenton-type catalyst based on iron salts (Fenton's reagent). ${ }^{[5]}$ However, this catalyst has some environmentally important drawbacks: ${ }^{[5]}$ i) stoichiometric amounts of iron are required, ii) production of hydroxyl radicals is limited to a $\mathrm{pH}=3$, iii) large quantities of acid are required to produce the optimal $\mathrm{pH}$, iv) a neutralization step of effluent and sludge disposal is required, v) several compounds in the wastewater make complexes with iron ions, which inhibits their activity and catalyzes side decomposition of $\mathrm{H}_{2} \mathrm{O}_{2}$, vi) removal of the catalyst from the system is difficult. The use of heterogeneous Fenton-type catalyst is a highly promising alternative, which could solve or minimize these problems caused by the presence of iron ions in the effluents, reduce recovery costs, and would also allow the use of conventional continuous-flow reactors at neutral $\mathrm{pH}$ and ambient temperatures. ${ }^{[6]}$ Heterogeneous Fenton-type nanocatalysts based on iron ${ }^{[7]}$ have therefore been intensively investigated in recent years. Nanoparticles are more efficient as conventional heterogeneous catalysts ${ }^{[8]}$ because of their large surface area and low diffusional resistance. Recent studies show that heterogeneous Fenton-type nanocatalysts based on manganese (nanosized $\mathrm{Mn}_{3} \mathrm{O}_{4}$ crystals) are also active towards the decomposition of $\mathrm{H}_{2} \mathrm{O}_{2}$ at room temperature. ${ }^{[9,10]}$

Herein we show for the first time that manganese functionalized silicate nanoparticles with interparticle porosity act as a superior Fenton-type catalyst in the WHPCO process at nearly neutral $\mathrm{pH}$ and at room temperature. X-ray absorption spectroscopic techniques (extended X-ray absorption fine structure 
- EXAFS and X-ray absorption near edge structure - XANES) demonstrate that the superior activity of the catalyst can be attributed uniquely to framework manganese. Moreover, we show that framework manganese within silicate nanoparticles, as oposed to manganese in manganese oxide nanoparticles $\mathrm{Mn}_{3} \mathrm{O}_{4}$ (Reference 10) and $\mathrm{Mn}_{2} \mathrm{O}_{3}$ (this study), does not promote the simultaneous decomposition of hydrogen peroxide used as the oxidizing agent.

\section{Results and Discussion}

We have prepared the heterogeneous Fenton-type nanocatalyst by incorporation of manganese into silicate nanoparticles of mesostructured KIL-2 with interparticle porosity, which was recently developed in our laboratory. ${ }^{[11]}$ Heterogeneous manganese Fenton-type catalysts with various Mn concentrations ( $\mathrm{Mn} / \mathrm{Si}$ ratios from 0.005 to 0.05 ) were prepared for the present study by direct two-step solvothermal synthesis (see details in Experimental Section). X-ray diffraction (XRD), scanning electron microscopy (SEM) and $\mathrm{N}_{2}$ adsorption iostherms confirmed the structural properties of the prepared catalyst (see Supporting Information and our previous report ${ }^{[11]}$ ). All prepared $\mathrm{Mn} /$ KIL-2 catalysts possess from 600 to $650 \mathrm{~m}^{2} / \mathrm{g}$ BET specific surface areas with pore diameters from 11 to $15 \mathrm{~nm}$ and silicate nanoparticles with typical dimensions between 10 and $20 \mathrm{~nm}$.

\subsection{Catalytic performance}

We have tested our catalysts in the WHPCO process, and the results are shown in Figure 1. Among MnKIL-based catalysts containing various loadings of manganese the lowest activity for methylene blue decomposition was observed for

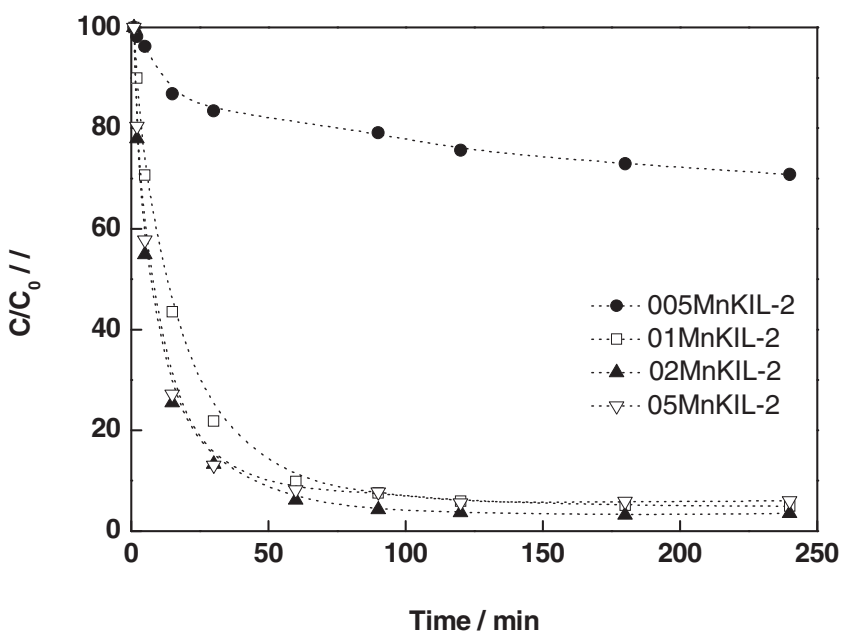

Figure 1. Relative concentration of methylene blue as a function of time obtained during the WHPCO carried out in a batch slurry reactor in the presence of manganese-containing silicate nanoparticles with $\mathrm{Si} / \mathrm{Mn}$ molar rations 0.005 (denoted as $005 \mathrm{MnKIL}-2$ ), 0.01 (denoted as $01 \mathrm{MnKIL}$ 2), 0.02 (denoted as $02 \mathrm{MnKIL}-2$ ) and 0.05 (denoted as 05MnKIL-2). Operating conditions: $\mathrm{T}=25^{\circ} \mathrm{C}$, atmospheric pressure, stirrer speed $320 \mathrm{rpm}$, $\mathrm{c}_{\text {cat. }}=400 \mathrm{mg} / \mathrm{L}, \mathrm{C}_{\mathrm{MB}, 0}=50 \mathrm{mg} / \mathrm{L}, \mathrm{C}_{\mathrm{H} 2 \mathrm{O} 2,0}=3.3 \mathrm{~g} / \mathrm{L}$.

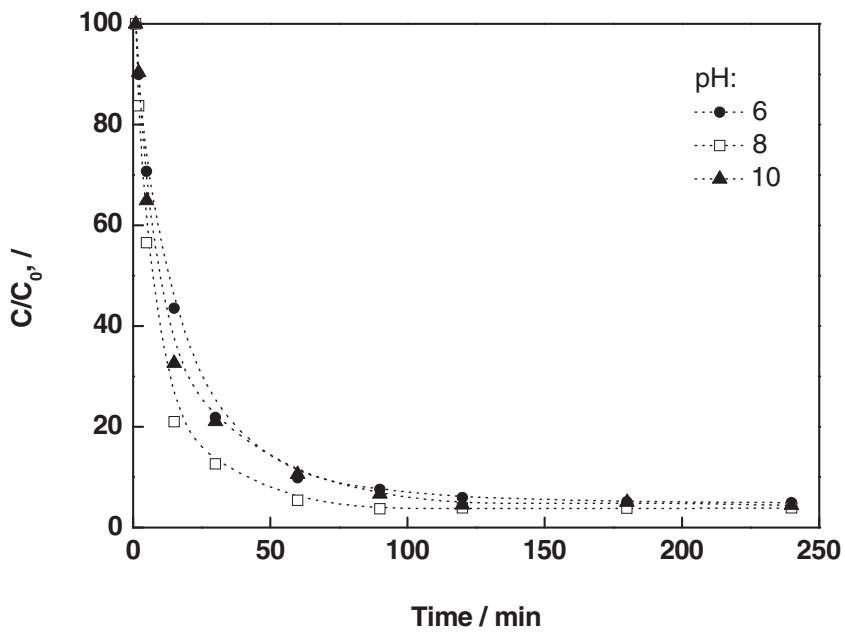

Figure 2. Relative concentration of methylene blue as a function of time obtained at different $\mathrm{pH}$ values in the presence of $01 \mathrm{MnKIL}-2$ catalyst. Operating conditions: $\mathrm{T}=25{ }^{\circ} \mathrm{C}$, atmospheric pressure, stirrer speed $320 \mathrm{rpm}, \mathrm{c}_{\text {cat. }}=400 \mathrm{mg} / \mathrm{L}, \mathrm{c}_{\mathrm{MB}, 0}=50 \mathrm{mg} / \mathrm{L}, \mathrm{C}_{\mathrm{H} 2 \mathrm{O} 2,0}=3.3 \mathrm{~g} / \mathrm{L}$.

the 005MnKIL-2 catalyst, while the 01MnKIL-2, 02MnKIL-2 and 05MnKIL-2 solids exhibited the highest activities for this reaction. A detailed examination of the aqueous-phase samples revealed, however, that the 02MnKIL-2 and 05MnKIL-2 catalysts significantly promote the simultaneous decomposition of hydrogen peroxide as an oxidizing agent. Less than $4 \%$ of the $\mathrm{H}_{2} \mathrm{O}_{2}$ initially present in the reaction suspension was consumed during the WHPCO process carried out in the presence of 01MnKIL-2 catalyst, while this value was found to be significantly higher and in the range of $30-100 \%$ for $02 \mathrm{MnKIL}-2$ and $05 \mathrm{MnKIL}-2$ materials. In this respect, 01MnKIL-2 solid was found as the optimal catalytic system, since it exhibits activity for methylene blue oxidation comparable to 02MnKIL-2 and 05MnKIL-2 catalysts along with a low tendency for decomposition of $\mathrm{H}_{2} \mathrm{O}_{2}$.

Acid/base properties of the catalyst surface could considerably affect heterogeneously catalyzed processes in the liquid phase. These effects were investigated by carrying out the oxidation of methylene blue in the presence of 01MnKIL-2 catalyst in a wide range of $\mathrm{pH}$ values $(\mathrm{pH}=6-10)$. It can be seen in Figure 2 that the $\mathrm{pH}$ value of the liquid phase exhibits a slight influence on the initial disappearance rates of methylene blue, which can be tentatively attributed to electrostatic repulsion between the reactant and differently charged catalyst surface. The final extent of the decomposition of methylene blue, however, is independent of the $\mathrm{pH}$ value of the solution.

The data listed in Table 1 clearly demonstrate that WHPCO of methylene blue carried out at mild reaction conditions in the presence of a series of MnKIL-2 catalysts results in deep oxidation of total organic carbon. The highest degree of TOC removal (more than $80 \%$ ) among the materials tested by us was obtained with the 01MnKIL-2 catalyst. Further, a comparison of relative concentration vs. time profile obtained in the presence of 005MnKIL-2 solid and TOC data listed in Table 1 confirms that low activity of this catalyst for methylene blue oxidation cannot be attributed to the adsorption of reaction intermediates 
Table 1. Total organic carbon (TOC) removal obtained during the WHPCO of aqueous solution of methylene blue in the presence of various catalysts.

\begin{tabular}{lcc}
\hline Catalyst & $\begin{array}{c}\text { TOC } \\
{[\mathrm{mg} / \text { L }]}\end{array}$ & $\begin{array}{c}\text { a) } \\
\text { TOC removal } \\
{[\%]}\end{array}$ \\
\hline Initial solution & 19.8 & 0 \\
$005 \mathrm{Mn} /$ KIL-2 & 11.7 & 40.8 \\
$01 \mathrm{Mn} / \mathrm{KIL}-2$ & 3.62 & 81.7 \\
$02 \mathrm{Mn} /$ KIL-2 & 4.84 & 75.5 \\
$05 \mathrm{Mn} /$ KIL-2 & 5.70 & 71.2 \\
b) $1 / 202 \mathrm{MnKIL-2}$ & 9.33 & 60.6 \\
\hline
\end{tabular}

a) Measured after the completion of oxidation run ( $\left.t=240 \mathrm{~min}) ;{ }^{b}\right) \mathrm{Half}$ amount of 02MnKIL-2 (50 mg).

on the catalyst surface (which could consequently result in catalyst deactivation due to blocking of active sites), since ultimate methylene blue conversion was very similar to the obtained TOC conversion after the completion of oxidation run (at $\mathrm{t}=$ $240 \mathrm{~min}$ ). In strong contrast to our result is the very low degree of TOC removal of less than $10 \%$, which was observed when WHPCO of methylene blue was carried out in the presence of $\mathrm{Mn}_{3} \mathrm{O}_{4}$ nanoparticles under similar reaction conditions. ${ }^{[12]} \mathrm{We}$ also note that only very low amounts of carbon (below $0.1 \mathrm{wt} . \%)$ were found to be deposited on the surface of the spent catalysts, which was confirmed by means of CHNS elemental analysis. Finally, a comparison of the data plotted in Figures 1 and 2 and listed in Table 1 confirms that various intermediates are accumulated in the liquid phase, most likely in the form of lowmolecular weight carboxylic acids (such as acetic acid), which are known to be resistant to further oxidation. ${ }^{[2 \mathrm{~d}, 2 \mathrm{e}]}$ This issue nonetheless remains to be investigated.

\subsection{Local environment of manganese}

The local structural environment of manganese in our catalyst materials was determined from XAS spectroscopic techniques XANES and EXAFS. The average oxidation number of manganese cations in $\mathrm{Mn} / \mathrm{KIL}-2$ samples with various concentrations of manganese was determined from XANES spectra. The position of manganese absorption edge shifts with the oxidation number of Mn atoms. A linear relationship between the shift of the edge and the valence state was established for atoms with the same type of ligands. ${ }^{[13]}$ For manganese atoms coordinated to oxygen a shift of $3.3 \mathrm{eV}$ per valence stateas has been established. ${ }^{[14]}$ The normalized Mn K-edge XANES data (Figure 3) of the 005MnKIL-2, 01MnKIL-2, 02MnKIL-2 and 05MnKIL-2 samples all exhibit edge shifts in the region between the Mn $\mathrm{K}$-edges of $\mathrm{MnO}$ and $\mathrm{Mn}_{2} \mathrm{O}_{3}$ reference compounds. The average oxidation states, therefore of $\mathrm{Mn}$ in these samples must be between $2+$ and $3+$.

If the similarity of the edge profiles allows, a comparison of the energy shift of the K-edge or pre-edge resonances in different samples can be used as an effective and precise tool to determine changes of the valence state of the atom. ${ }^{[13 a, 15-17]}$ However, when the edge profiles are significantly different, due to different local structures around investigated cations,

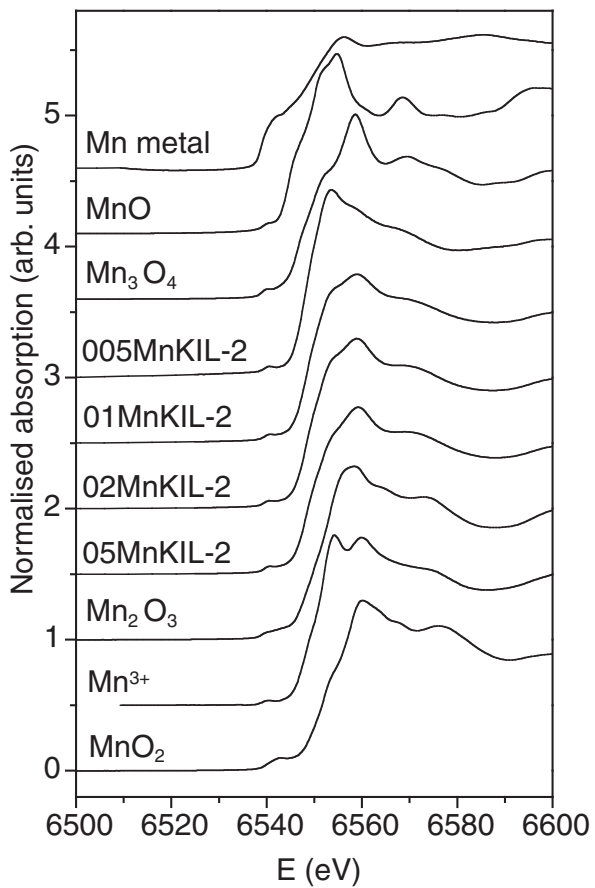

Figure 3. Normalized Mn K-edge XANES data of MnKIL-2 samples and $\mathrm{Mn}$ reference samples ( $\mathrm{Mn}$ metal, $\mathrm{MnO}, \mathrm{Mn}_{3} \mathrm{O}_{4}, \mathrm{~K}_{3}\left[\mathrm{Mn}^{3+}\left(\mathrm{C}_{2} \mathrm{O}_{4}\right)_{3}\right] \cdot 3 \mathrm{H}_{2} \mathrm{O}$, $\mathrm{Mn}_{2} \mathrm{O}_{3}$ and $\mathrm{MnO}_{2}$ ).

the comparison of the edge shift or the shift of the pre-edge resonances is hindered. ${ }^{[18,19]}$ Among different approaches to precisely determine average valence state of the atom in the sample from XANES data, ${ }^{[16,17]}$ best results are obtained by a linear combination fit with XANES data of proper reference compounds with known valence states of the element, with similar symmetry, same type of neighbour atoms in nearest coordination shells, arranged in a similar local structure. ${ }^{[18,20]}$ If the sample contains same cation in two or more sites with different local structures and valence state, then the measured XANES data is a linear combination of individual XANES data of different cation sites. In such cases linear combination fit is especially precise in determination of the relative amounts of the cation at each site. ${ }^{[21]}$ In our case we chose $\mathrm{MnO}$ as reference for $\mathrm{Mn}^{2+}$ and and $\mathrm{Mn}_{2} \mathrm{O}_{3}$ as reference compounds for $\mathrm{Mn}^{3+}$. Although the two reference compounds do not posess exactly the same Mn site simetry as Mn in our samples, the similarity of the Mn edge profiles alowed to obtain good linear combination fits (as shown in the Supporting Information in the Figure S4-1). The average valence states of $\mathrm{Mn}$ in the samples obtained by this procedure are presented in Table 2 .

Table 2. Average oxidation numbers of the MnKIL-2 samples determined from the Mn K-edge shift. (Uncertainties for all valence states are 0.1.)

\begin{tabular}{ccccc}
\hline & $\mathrm{Mn} / \mathrm{Si}$ & $\mathrm{Mn} / \mathrm{Si}$ & $\mathrm{Mn} / \mathrm{Si}$ & $\mathrm{Mn} / \mathrm{Si}$ \\
\cline { 2 - 5 } & 0.005 & 0.01 & 0.02 & 0.05 \\
catalyst $^{\mathrm{a}}{ }^{2}$ & $2.4+$ & $2.7+$ & $2.8+$ & $2.9+$
\end{tabular}

a) Catalyst before the Fenton reaction. 


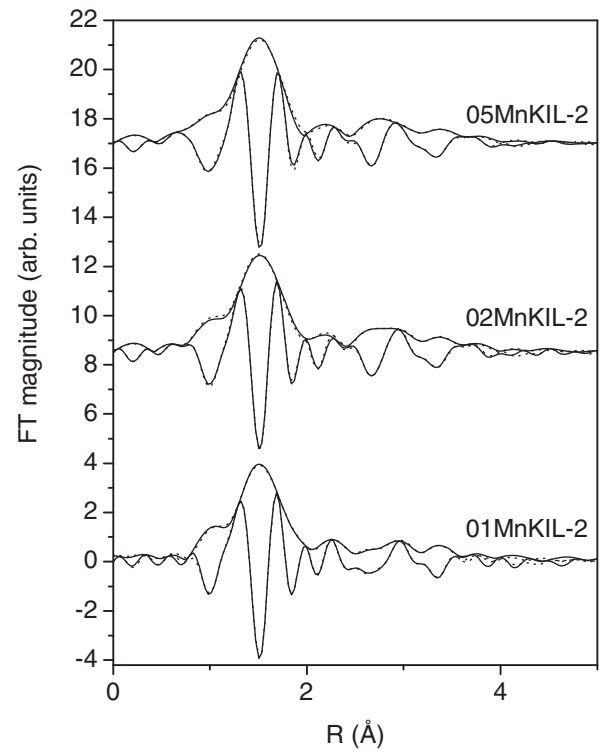

Figure 4. Fourier transform magnitudes and imaginary parts of the $k^{3}$ weighed Mn EXAFS data of Mn/KIL-2 samples (solid line-experiment, dotted line-EXAFS model).

We note that the average valence state of $\mathrm{Mn}$ atoms in the sample increases with concentration of manganese in the sample. The sample with the lowest concentration of $\mathrm{Mn}$ (005MnKIL-2) has an average oxidation number of 2.4+, which corresponds to $\mathrm{Mn}^{2+}$ to $\mathrm{Mn}^{3+}$ ratio of $60 \% / 40 \%$. The ratio for the $01 \mathrm{MnKIL}-2$ sample is decreased to $30 \% / 70 \%$, while that for the sample with the highest Mn loading (05MnKIL-2) reaches $10 \% / 90 \%$. This coexistence of $\mathrm{Mn}^{2+}$ and $\mathrm{Mn}^{3+}$ in different molar ratio is in agreement with previous studies on $\mathrm{Mn}$ incorporation into porous silicates..$^{[9,11]}$ In Mn-funcionalized silicates during the synthesis process first $\mathrm{Mn}^{2+}$ is incorporated up to the certain limit (005MnKIL-2 contains more $\mathrm{Mn}^{2+}$ as $01 \mathrm{MnKIL}-2)$, than $\mathrm{Mn}^{3+}$ is incorporated up to the certain limit (01MnKIL-2 contains more $\mathrm{Mn}^{3+}$ as 005MnKIL-2) and only after that $\mathrm{Mn}$ cations begin to incorporate as extra-framework $\mathrm{Mn}^{3+}$ (02MnKIL-2 and 05MnKIL-2). Since XANES can only detect the average valence state of all $\mathrm{Mn}$ atoms in the sample, we cannot completely exclude the possibility of the presence of some $\mathrm{Mn}^{4+}$ ions. It is very likely that when manganese oxides are present in the sample, they are in the form of various Mn oxides, and these would be a mixture of various nano-crystalline forms. Finally, it might be speculated that in comparison to other solids low activity of 005MnKIL-2 catalyst for methylene blue degradation (Figure 1) might be attributed to inappropriate $\mathrm{Mn}^{2+} / \mathrm{Mn}^{3+}$ molar ratio, which in the given range of operating conditions doesn't allow optimal redox behavior of this solid during the entire reaction course. However, this remains to be further investigated.

Mn K-edge EXAFS data of the 01MnKIL-2, 02MnKIL-2 and 05MnKIL-2 samples along with the best fit EXAFS models (Figure 4) give a description of the short-range order of the manganese atoms in terms of neighbors, distances and thermal and static disorder (see details in Experimental Section). A complete list of these best fit parameters is given in Table 3 .
Table 3. Structural parameters of the nearest coordination shells around $\mathrm{Mn}$ atom in the MnKIL-2 samples: type of neighbor atom, average number $\mathrm{N}$, distance $\mathrm{R}$, and Debye-Waller factor $\sigma^{2}$. The amplitude reduction factor $\left(\mathrm{S}_{0}^{2}=0.80 \pm 0.05\right)$ was determined on $\mathrm{MnO}$ and kept fixed during the fit. Uncertainties in the last digit are given in the parentheses.

\begin{tabular}{|c|c|c|c|}
\hline \multirow{2}{*}{$\begin{array}{l}01 \text { MnKIL-2 } \\
\text { Neighbor }^{\text {a) }} \\
\end{array}$} & \multirow[b]{2}{*}{$\mathrm{N}$} & \multirow[b]{2}{*}{$\mathrm{R}[\AA ̊]$} & \multirow[b]{2}{*}{$\sigma^{2}\left[\AA^{2}\right]$} \\
\hline & & & \\
\hline $\mathrm{O}$ & $1.8(2)$ & $1.891(4)$ & $0.0033(9)$ \\
\hline $\mathrm{O}$ & $0.4(2)$ & $2.21(2)$ & $0.0033(9)$ \\
\hline $\mathrm{O}$ & $0.9(2)$ & $2.83(1)$ & 0.006 \\
\hline $\mathrm{Si}$ & $3(1)$ & $3.35(1)$ & $0.018(6)$ \\
\hline O & $1.4(4)$ & $3.71(2)$ & 0.006 \\
\hline \multicolumn{4}{|l|}{ 02MnKIL-2 } \\
\hline Neighbor & $N$ & $R(\AA ̊)$ & $\sigma^{2}\left(\AA^{2}\right)$ \\
\hline O & 3 & $1.897(2)$ & $0.0065(3)$ \\
\hline O & 2 & $2.198(9)$ & $0.0065(3)$ \\
\hline O & 1 & $2.41(2)$ & $0.0065(3)$ \\
\hline $\mathrm{Mn}$ & $7(3)$ & $3.52(3)$ & $0.017(4)$ \\
\hline O & $6(1)$ & $3.64(3)$ & 0.007 \\
\hline $\mathrm{Mn}$ & $4(2)$ & $3.79(2)$ & $0.017(4)$ \\
\hline \multicolumn{4}{|l|}{ 05MnKIL-2 } \\
\hline Neighbor & $\mathrm{N}$ & $R(\AA ̊)$ & $\sigma^{2}\left(\AA^{2}\right)$ \\
\hline O & 3 & $1.903(3)$ & $0.0064(3)$ \\
\hline O & 2 & $2.22(1)$ & $0.0064(3)$ \\
\hline O & 1 & $2.43(2)$ & $0.0064(3)$ \\
\hline $\mathrm{Mn}$ & $7(3)$ & $3.52(3)$ & $0.017(5)$ \\
\hline $\mathrm{O}$ & $6(1)$ & $3.64(3)$ & 0.007 \\
\hline $\mathrm{Mn}$ & $3(2)$ & $3.79(3)$ & $0.017(5)$ \\
\hline
\end{tabular}

a) The type of the neighboring atom, average number $N$, distance $R$ and the DebyeWaller factor $\sigma^{2}$. Uncertainties in the last digit are given in the parentheses.

The manganese cations in $\mathrm{Mn} / \mathrm{KIL}-2$ with $\mathrm{Mn} / \mathrm{Si}=0.01$ (denoted 01MnKIL-2) were found to be coordinated to three oxygen atoms in the first coordination shell, two of them are at a shorter distance of $1.9 \AA$ and one of them at a longer distance of $2.2 \AA$. The short distance is consistent with the average tetrahedral $\mathrm{Mn}^{+3}$-O distance of $1.93(4) \AA$ reported for $\mathrm{MnAsO}_{4} \cdot{ }^{[22]} \mathrm{It}$ is also consistent with the distances found in other manganese porous silicates (MnS-1, MnMCM-41, MnMCM-48, MnTUD-1) which contain mainly framework $\mathrm{Mn}^{+3} .^{[9]}$ We can therefore conclude that the Mn cations in 01MnKIL-2 are coordinated to three framework oxygen atoms in a distorted and coordinatively unsaturated 3-fold symmetry, which is characteristic of Lewis acid sites. In the second coordination sphere we find silicon atoms at the distance of $3.35 \AA$. The result suggests that manganese is indeed incorporated into the silicate framework, which is indirectly in a good agreement with HRTEM investigations, where no manganese oxide particles were visible on the surface (Figure 5).

The manganese cations in the Mn/KIL-2 samples with $\mathrm{Mn} /$ Si ratio 0.02 (denoted 02MnKIL-2) and with $\mathrm{Mn} / \mathrm{Si}$ ratio 0.05 (denoted 05MnKIL-2) are found to be coordinated to six oxygen 


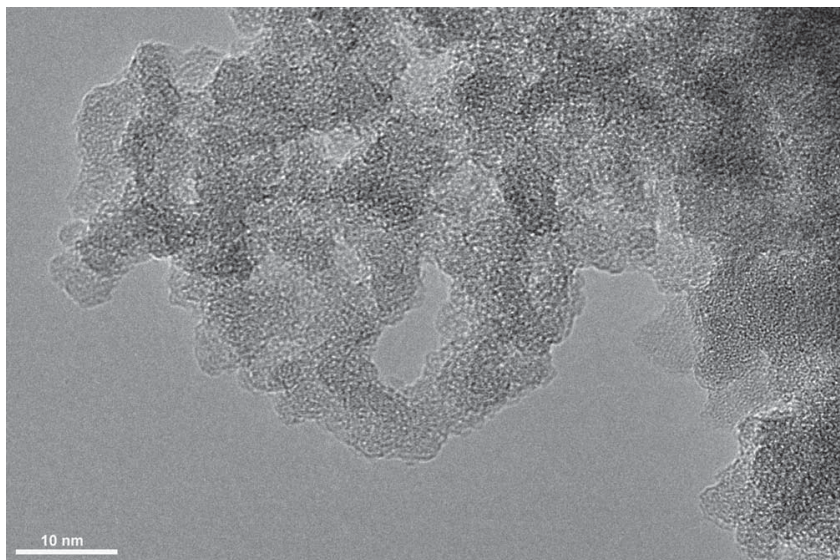

Figure 5. HRTEM image of $01 \mathrm{MnKIL-2}(\mathrm{Mn} / \mathrm{Si}=0.01)$ with silicate nanoparticles with typical dimensions between 10 to $20 \mathrm{~nm}$.

atoms in the first coordination shell. The distances are consistent with the octahedral $\mathrm{Mn}^{+3}$-O bond lengths in $\mathrm{Mn}_{2} \mathrm{O}_{3}$, which range from 1.91 to $2.22 \AA .{ }^{[23]} \mathrm{Mn}$ atoms are found in the second coordination sphere, which clearly indicates the presence of manganese oxides in these samples. The result is in good agreement with HRTEM results, where extra-framework manganese oxides in the frame of fibre-like shapes are visible on the surface (Figure 6 and Supporting Information). We confirmed that these crystallites have $\mathrm{Mn}_{2} \mathrm{O}_{3}$ bixbyite structure (see Supporting Information) from selected-area electron diffraction (SAED).

\subsection{Hypothesis}

The catalytic performance of manganese-functionalized silicate nanoparticles along with structural results from XAS studies of the local environment indicate that manganese is incorporated in the framework for materials with $\mathrm{Mn} / \mathrm{Si}$ ratio up to a certain value (about 0.01 ). When the manganese concentration exceeds

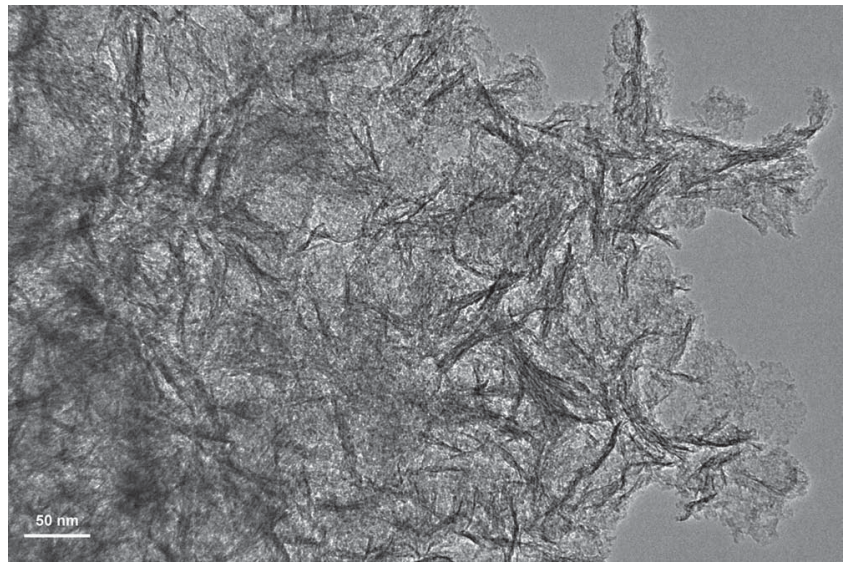

Figure 6. HRTEM image of $05 \mathrm{MnKIL}-2(\mathrm{Mn} / \mathrm{Si} \geq 0.05)$ with silicate nanoparticles 10 to $20 \mathrm{~nm}$ in size and with additional extra-framework needlelike $\mathrm{Mn}_{2} \mathrm{O}_{3}$ oxide nanoparticles around $20 \mathrm{~nm}$ in size.

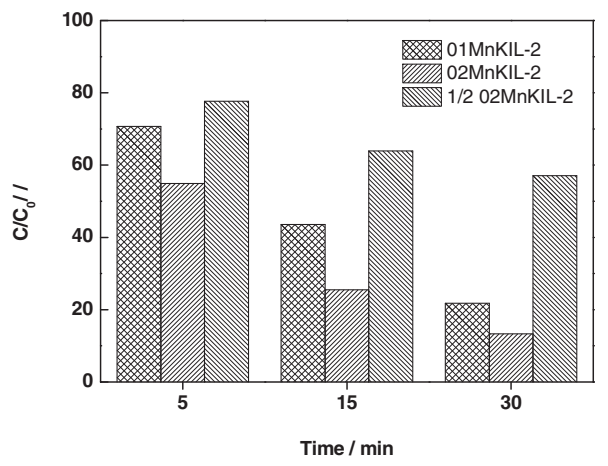

Figure 7. Relative concentration of methylene blue as a function of time obtained during the WHPCO process carried out at $\mathrm{T}=25^{\circ} \mathrm{C}$ and atmospheric pressure at various catalyst loadings.

this value, the excess $\mathrm{Mn}$ is deposited in the form of Mn oxide nanoparticles on the surface of the samples. This would mean that the 01MnKIL-2, 02MnKIL-2 and 05MnKIL-2 samples all have the same amount of manganese incorporated in the framework. If we assume that only framework manganese was catalytically active, the lack of increase in the catalytic performance of samples with higher Mn content, i.e., 02MnKIL-2 and $05 \mathrm{MnKIL}-2$, can be rationalized. We tested this hypothesis by performing a catalytic reaction with the 02MnKIL-2 catalyst with only one half of the sample loading typically used in the WHPCO process (Table 1). In this case the total amount of manganese in the reaction would be equal to that in the 01MnKIL-2 sample. If all the manganese in this sample were active, the rate of the reaction should thus be the same as with the 01MnKIL-2 sample. If on the other hand only the framework manganese was catalytically active, the rate of discoloration of the dye solution should be much slower for 02MnKIL-2. The result of the catalytic test done by using one half of the 02MnKIL-2 sample (Figure 7) clearly supports the latter hypothesis.

A comparison of data depicted in Figure 7 which were obtained with various loadings of 02MnKIL-2 catalyst, shows that within an experimental error conversion of methylene blue increases linearly with an increase of catalyst concentration. This finding confirms that WHPCO runs were in the given range of operating conditions performed in the kinetic regime. This decrease in catalytic performance when a smaller amount of 02MnKIL-2 sample is used, is thus a conclusive proof that there is an upper limit to the amount of manganese that can be incorporated in the framework structure. This supports our previous conclusions on the incorporation limit of manganese into silicates. ${ }^{[9]}$ It also confirms that only framework manganese is catalytically active in the sense of producing reactive hydroxyls. Mn oxide nanoparticles on the catalyst surface, on the other hand, preferentially promote liquid-phase $\mathrm{H}_{2} \mathrm{O}_{2}$ decomposition and do not contribute to the decomposition of methylene blue. This is in agreement with the results in the reference 10 .

\section{Conclusions}

We have demonstrated that among porous silicate catalysts containing various loadings of manganese high catalytic activities 
for methylene blue decomposition, as a model organic pollutant in the WHPCO process occur in the presence of catalysts with $\mathrm{Mn} / \mathrm{Si}=0.01,0.02$ and 0.05 (Figure 1, 02MnKIL-2, 02MnKIL-2 and 05MnKIL-2) in the reaction suspension, while lower catalytic activity is observed in a presence of catalyst with $\mathrm{Mn} / \mathrm{Si}$ $=0.005$ (Figure 1, 005MnKIL). Catalysts with extra-framework manganese (Figure 1, 02MnKIL-2 and 05MnKIL-2), however, were found by a detailed examination of aqueous-phase samples to significantly promote simultaneous decomposition of hydrogen peroxide used as the oxidizing agent. In view of the above results we can conclude that the catalyst with framework manganese and molar ratio $\mathrm{Si} / \mathrm{Mn}=0.01$ (Figure 1, 01MnKIL-2) to be the optimal catalytic system, since it exhibits both high activity for methylene blue oxidation, comparable to catalysts with extra-framework manganese, but also a low tendency for $\mathrm{H}_{2} \mathrm{O}_{2}$ decomposition.

Our results clearly demonstrate that these materials facilitate deep oxidation of organic matter towards carbon dioxide under mild reaction conditions ( $80 \%$ in 30 minutes), which makes them highly promising catalysts for AOP wastewater treatment.

\section{Experimental Section}

Synthesis: We incorporate manganese with $\mathrm{Mn} / \mathrm{Si}$ molar rations from 0.005 to 0.05 via two-step solvothermal synthesis into a new inorganic silicate matrice from KIL family (KIL-2). KIL-2 is a mesostructured silicate formed from silicate nanoparticles (10-20 nm in size) with interparticle porosity. ${ }^{11]}$ We denoted the potential catalysts as $005 \mathrm{MnKIL}-2(\mathrm{Mn} / \mathrm{Si}=0.005), 01 \mathrm{MnKIL}-2$ $(\mathrm{Mn} / \mathrm{Si}=0.01), 02 \mathrm{MnKIL}-2 \quad(\mathrm{Mn} / \mathrm{Si}=0.02)$ and $05 \mathrm{MnKIL}-2$ $(\mathrm{Mn} / \mathrm{Si}=0.05)$. In the first step, manganese acetate tetrahydrate $\left(\mathrm{Mn}\left(\mathrm{CH}_{3} \mathrm{COO}\right)_{2} \cdot 4 \mathrm{H}_{2} \mathrm{O}\right.$, Aldrich), tetraethyl ortosilicate (98\% TEOS, Acros) and triethanolamine (99\% TEA, Fluka) were stirred for $30 \mathrm{~min}$. Finally demineralised water was added to the above mixture, followed by the addition od tetraethylammonium hydroxide $(20 \%$ $\mathrm{TEAOH}$, Acros). The solution was mixed with magnetic stirrer to obtain homogeneous gel. The final gels, with molar compositions of TEOS: 0.5 TEA: (0.005-0.05) $\mathrm{MnO}: 0.1 \mathrm{TEAOH}: 11 \mathrm{H}_{2} \mathrm{O}$ were aged overnight at room temperature and then dried in an oven for $24 \mathrm{~h}$ at $50{ }^{\circ} \mathrm{C}$. In the second step, the gel was solvothermally treated in ethanol in Teflon-lined stainless steel autoclaves at $150{ }^{\circ} \mathrm{C}$ for $48 \mathrm{~h}$. Removal of the template was performed by calcination at $500^{\circ} \mathrm{C}$ for $10 \mathrm{~h}$ using a ramp rate of $1{ }^{\circ} \mathrm{C} / \mathrm{min}$ in the flow of air. In this way the thermal stability of the product was also proved. The hydrothermal stability of the products was verified by keeping them in the boiling water for $2 \mathrm{~h}$.

Characterization: Basic structural characterization of prepared catalysts was performed using X-ray powder diffraction (XRD), $\mathrm{N}_{2}$ sorption measurements and scanning electron microscopy (SEM). A more detailed description of the characterization is given in Supporting Information and Reference 11. Mesostructure characteristics, the presence of manganese oxide nanoparticles and their structural correlation with mesoporous matrix were investigated by highresolution transmission electron microscopy (HRTEM). The analysis was performed on a 200-kV field-emission gun (FEG) microscope JEOL JEM 2100. For HRTEM studies a drop of an ethanol diluted nanoparticles solution was placed on a copper grid and dried at room temperature. The specimens were additionally coated with carbon in order to prevent excessive charging and decomposition of the sample under the electron beam.

Local environment of manganese was determined using X-ray absorption spectroscopy (XAS). XAS spectra of the samples and $M n$ reference compounds were measured in the energy region of the $\mathrm{Mn}$
K-edge in transmission detection mode at beamline $\mathrm{C}$ of HASYLAB synchrotron radiation facility at DESY in Hamburg. A Si(111) double crystal monochromator was used with about $1 \mathrm{eV}$ energy resolution at the $\mathrm{Mn}$ K-edge $(6539 \mathrm{eV})$. Higher harmonics were effectively eliminated by a slight detuning of the second monochromator crystal, keeping the intensity at $60 \%$ of the rocking curve with the beam stabilization feedback control. The intensity of the monochromatic X-ray beam was measured by three consecutive ionization chambers filled with 960 mbar $N_{2}, 315$ mbar Ar and 960 mbar Ar for the first, second and third ionization chamber, respectively. The samples and the reference compounds were prepared as homogeneous self-supporting pellets with the total absorption thickness $(\mu \mathrm{d})$ of about 2.5 above the $\mathrm{Mn}$ K-edge. The total absorption thickness of 2.5 was chosen to improve signal to noise ratio in EXAFS spectrum. To prevent systematic errors due to inhomogeneity in the sample, which are more expressed in the case of thick samples, we took special care to prepare homogenous samples. The pellets were mounted on a sample holder in a vacuum chamber of the beamline, so that they were kept in high vacuum during the measurements. The absorption spectra of the samples were measured within the interval $[-190 \mathrm{eV}, 960 \mathrm{eV}]$ relative to the $\mathrm{Mn}$ K-edge. In the XANES region equidistant energy steps of $0.3 \mathrm{eV}$ were used, while for the EXAFS region equidistant $k$-steps $\left(k=0.03 \AA^{-1}\right)$ were adopted with the integration time of $1 \mathrm{~s} / \mathrm{step}$. Exact energy calibration was established with the simultaneous absorption measurements on $\mathrm{Mn}$ metal foil inserted between the second and third ionization cell. $\mathrm{Mn}$ K-edge EXAFS data were quantitatively analyzed for the numbers, distances and Debye-Waller factors for the nearest coordination shells of neighbor atoms. The quantitative analysis of the $\mathrm{k}^{3}$-weighted $\mathrm{Mn}$ EXAFS data was performed with the IFEFFIT program packages ${ }^{[24]}$ using FEFF6 code $^{[20]}$ in the $\mathrm{k}$ range of $3.5-12.3 \AA^{-1}$ for $05 \mathrm{MnKIL}-2$ and 02MnKIL-2 samples and in the range of 3.3-12.3 $\AA^{-1}$ for the $01 \mathrm{MnKIL}-2$ sample.

Catalysis: WHPCO studies were carried out in a 250-ml five-necked glass reactor equipped with a magnetic stirrer, heating jacket and a condenser. In a typical run, water containing methylene blue dye $\left(c_{0}=\right.$ $50 \mathrm{mg} / \mathrm{L}$ ) was transferred into the reactor and a known amount of a catalyst $(100 \mathrm{mg}$ ) was added to the solution. The suspension was thermostated at $\mathrm{T}=25{ }^{\circ} \mathrm{C}$ under continuous stirring and sparging by means of pure nitrogen. Just prior starting the reaction, concentrated solutions of hydrogen peroxide were added. Representative aqueousphase samples were withdrawn at periodic intervals (up to $240 \mathrm{~min}$ ) and immediately filtered $(0.8 \mu \mathrm{m})$. Reactions were also performed in the absence of oxidant or catalyst. A pH range of 6-10 was used to study the reaction. The initial $\mathrm{pH}$ was adjusted with $5.0 \mathrm{~m} \mathrm{NaOH}$ or $\mathrm{HCl}$ aqueous solutions using a Metrohm pH meter (model 781) equipped with a combined $\mathrm{pH}$ electrode. The $\mathrm{pH}$ meter was calibrated using standard buffer solutions purchased from Merck before every measurement. Temporal concentration of dye in withdrawn samples was determined by means of UV/vis spectrophotometry. Concentration of $\mathrm{H}_{2} \mathrm{O}_{2}$ in withdrawn liquid-phase samples was determined by redox titration with a standardized potassium permanganate solution using an automatic titrator (Mettler Toledo, model T50). The total amount of organic substances in withdrawn aqueous-phase samples was determined by measuring the total organic carbon (TOC). The latter was determined applying a high-temperature catalytic oxidation (HTCO) method by using a Rosemount/Dohrmann DC-190 TOC analyzer equipped with a NDIR detector, by subtracting measured inorganic carbon (IC) content from measured total carbon (TC) content. In all analyses, 3-4 repeated measurements were taken for each liquid-phase sample, and the average value of TOC was reported. The error of analysis was never greater than $\pm 0.5 \%$

\section{Supporting Information}

Supporting Information is available from the Wiley Online library or from the author. 


\section{Acknowledgements}

Financial support from the Slovenian Research Agency, EN-FIST Centre of Excellence and DESY and EU 7. Framework Programme (FP7/2007. 2013) ELISA ( ${ }^{\circ}$ 226716), and access to SR facilities of HASYLAB (project II-20080058 EX) are acknowledged.

Received: October 2, 2011

Revised: November 7, 2011

Published online:

[1] a) P. Burhardt-Holm, Int. J. Water Resour. Dev. 2010, 26, 477; b) B. O. Clarke, S. R. Smith, Env. Int. 2011, 37, 226.

[2] a) K. Singh, S. Arora, Crit. Rev. Environ. Sci. Technol. 2011, 41, 807; b) T. Y. Chiu, N. Paterakis, E. Cartmell, M. D. Scrimshaw, J. N. Lester, Crit. Rev. Environ. Sci. Technol. 2010, 40, 199; c) K. H. Kim, S. K. Ihm, J. Hazard. Mater. 2011, 186, 16; d) J. Levec, A. Pintar, Catal. Today 2007, 124, 172; e) A. Pintar, Catal. Today 2003, 77, 451; f) K. Pirkanniemi, M. Sillanpää, Chemosphere 2002, 48, 1047.

[3] A. S. Stasinakis, Global NEST J. 2008, 10, 376

[4] S. Parathoner, G. Centi, Top. Catal. 2005, 33, 207.

[5] S. Caudo, G. Centi, C. Genovese, S. Parathoner, Top. Catal. 2006, 40, 207.

[6] a) J.J. Pignatello, E. Oliveros, A. MacKay, Crit. Rev. Env. Sci. Technol. 2006, 36, 1; b) S. Nevalon, M. Alvaro, H. Garcia, Appl. Catal., B 2010, 99, 1; c) M. Hartmann, S. Kullmann, H. Keller, J. Mater. Chem. 2010, 20, 9002.

[7] E. G. Garrido-Ramírez, B. K. G. Theng, M. L. Mora, Appl. Clay Sci. 2010, 47, 182.

[8] a) A. T. Bell, Science 2003, 299, 1688; b) R. Schlögl, S. B. Abd Hamid, Angew. Chem., Int. Ed. 2004, 43, 1628; c) T. Valdés-Solís, P. Valle-Vigón, S. Álvares, G. Marbán, A. B. Fuertes, Catal. Commun. 2007, 8, 2037.

[9] N. Novak Tušar, S. Jank, R. Gläser, ChemCatChem 2011, 3, 254.

[10] T. Rhadfi, J.-Y. Piquemal, L. Sicard, F. Herbst, E. Briot, M. Benedetti, A. Atlamsani, Appl. Catal., A 2010, 386, 132.

[11] N. Novak Tušar, A. Ristić, G. Mali, M. Mazaj, I. Arčon, D. Arčon, V. Kaučič, N. Zabukovec Logar, Chem.- Eur. J. 2010, 16, 5783.

[12] T. Rhadfi, J.-Y. Piquemal, L. Sicard, F. Herbst, E. Briot, M. Benedetti, A. Atlamsani, Appl. Catal., A 2010, 386, 132.

[13] a) J. Wong, F. W. Lytle, R. P. Messmer, D. H. Maylotte, Phys. Rev. B 1984, 30, 5596-5610; b) I. Arčon, B. Mirtič, A. Kodre, J. Am. Ceram. Soc. 1998, 81, 222.

[14] a) T. Ressler, J. Wong, J. Roos, J. Synchrotron Rad. 1999, 6, 656-658; b) N. Novak Tušar, N. Zabukovec Logar, G. Vlaic, I. Arčon, D. Arčon, N. Daneu, V. Kaučič, Micropor. Mesopor. Mater. 2005, 82, 129.

[15] I. Arčon, A. Benčan, A. Kodre, M. Kosec, X-ray Spectrom. 2007, 30, 301.

[16] F. Farges, Phys. Rev. B. 2005, 71, 1551091-14.

[17] a) F. Farges, G. E. Brown, J. J. Rehr, Phys. Rev. B. 1997, 56, 1809; b) J.-P. Rueff, L. Journel, P.-E. Petit, F. Farges, Phys. Rev. B 2004, 69, 2351071-9.

[18] I. Arčon, J. Kolar, A. Kodre, D. Hanžel, M. Strlič, X-ray Spectrom. 2007, 36, 199

[19] A. Pantelouris, H. Modrow, M. Pantelouris, J. Hormes, D. Reinen, Chem. Phys. 2004, 13, 300.

[20] R. Ravel, M. Newville, J. Synchrotron Rad. 2005, 12, 537.

[21] R. Dominko, I. Arčon, A. Kodre, D. Hanzel, M. Gaberšček, J. Power Sources 2009, 189, 51.

[22] M. A. G. Aranda, J.-P. Attfield, S. Bruque, Inorg. Chem. 1993, 32, 1925.

[23] X. Wang, G. Li, Q. Chu, X. Liu, S. Feng, Chem. J. Chin. Univ. 2007, 28,821 .

[24] J. J. Rehr, R. C. Albers, S. I. Zabinsky, Phys. Rev. Lett. 1992, 69, 3397. 